\title{
Exploring the Kondo effect of an extended impurity with chains of Co adatoms in a magnetic field
}

\author{
Bimla Danu, ${ }^{1, *}$ Fakher Assaad ${ }^{2,3, \dagger}$ and Frédéric Mila ${ }^{1, \ddagger}$ \\ ${ }^{1}$ Institute of Physics, École Polytechnique Fédérale de Lausanne (EPFL), CH-1015 Lausanne, Switzerland \\ ${ }^{2}$ Institut für Theoretische Physik und Astrophysik, \\ Universität Würzburg, Am Hubland, D-97074 Würzburg, Germany \\ ${ }^{3}$ Würzburg-Dresden Cluster of Excellence ct.qmat
}

(Dated: March 21, 2019)

\begin{abstract}
Motivated by recent STM experiments, we explore the magnetic field induced Kondo effect that takes place at symmetry protected level crossings in finite Co adatom chains. We argue that the effective two-level system realized at a level crossing acts as an extended impurity coupled to the conduction electrons of the substrate by a distribution of Kondo couplings at the sites of the chain. Using auxiliary-field quantum Monte Carlo simulations, which quantitatively reproduce the field dependence of the zero-bias signal, we show that a proper Kondo resonance is present at the sites where the effective Kondo coupling dominates. Our modeling and numerical simulations provide a theoretical basis for the interpretation of the STM spectrum in terms of level crossings of the Co adatom chains.
\end{abstract}

PACS numbers: 72.15.Qm,75.20.Hr,75.10.Pq,75.30.Hx

The Kondo effect is one of the most extensively studied and adequately addressed many-body process occurring due to the screening of a local moment by a conduction electron cloud $^{1-3}$. On the experimental side, recent advances in scanning tunneling microscopy (STM) open greater opportunities to realise and investigate the Kondo effect in various Kondo nanostructures ${ }^{4-11}$. For instance, the recent STM experiments on finite atomic spin-chain realizations of Co adatoms in the presence of an external magnetic field have revealed an interesting interplay between the Kondo problem and the physics of quantum spin chains in a field ${ }^{11}$. Co adatoms on $\mathrm{Cu}_{2} \mathrm{~N} / \mathrm{Cu}(100)$ surface carry a spin-3/2 with a strong uniaxial hard-axis anisotropy $(D)^{10,12}$, and applying an external magnetic field perpendicular to the surface, the Co adatom chain effectively behaves like a spin- $1 / 2 \mathrm{XXZ}$ chain in transverse field. The magnetic field induced level crossings of finite XXZ and SU(2) chains are similar so that the experiments of Ref. [11] can be discussed in the context of the $\mathrm{SU}(2)$ invariant version of the model:

$$
\begin{aligned}
\hat{H}= & -t \sum_{\langle\boldsymbol{i}, \boldsymbol{j}\rangle, \sigma}\left(\hat{c}_{\boldsymbol{i}, \sigma}^{\dagger} \hat{c}_{\boldsymbol{j}, \sigma}+h . c\right)+J_{k} \sum_{\boldsymbol{l}=1}^{L} \hat{\boldsymbol{S}}_{\boldsymbol{l}}^{c} \cdot \hat{\boldsymbol{S}}_{\boldsymbol{l}} \\
& +J_{h} \sum_{\boldsymbol{l}=1}^{L-1} \hat{\boldsymbol{S}}_{\boldsymbol{l}} \cdot \hat{\boldsymbol{S}}_{\boldsymbol{l}+\Delta \boldsymbol{l}}-g \mu_{B} h^{\mathbf{z}} \sum_{\boldsymbol{l}=1}^{L} \hat{S}_{\boldsymbol{l}}^{z} .
\end{aligned}
$$

Here, $t$ is the hopping parameter of the conduction electrons, $J_{k}$ the antiferromagnetic Kondo coupling between a Co adatom and the conduction electrons, $J_{h}$ the Heisenberg antiferromagnetic coupling, $h^{\mathbf{z}}$ an external magnetic field in the $z$ direction, $L$ the length of the Heisenberg chain, $\hat{\boldsymbol{S}}_{\boldsymbol{l}}$ spin-1/2 operators and $\hat{\boldsymbol{S}}_{\boldsymbol{l}}^{c}=$ $\frac{1}{2} \sum_{\sigma, \sigma^{\prime}} \hat{c}_{\boldsymbol{l}, \sigma}^{\dagger} \boldsymbol{\sigma}_{\sigma, \sigma^{\prime}} \hat{c}_{\boldsymbol{l}, \sigma^{\prime}}$ denotes the spin of conduction electrons. Throughout the calculation we set $t, \mu_{B}=1$ and $g=2$.

When the Kondo coupling is switched off $\left(J_{k}=0\right)$, the chain undergoes a series of level crossings that lead to steps in the magnetization curve. In the geometry used in the experiment of Ref. [11], $J_{h}$ and the Kondo energy $\epsilon_{k}=k_{B} T_{k}$ are both of the order of $0.2 \mathrm{meV}$, with two important consequences: There is a competition between the Heisenberg coupling and the Kondo effect, and one can reach the saturation field of the isolated chain.

The main result of the STM experiments of Ref. [11] is to demonstrate that the differential conductance exhibits a series of anomalies as a function of the field, and that these anomalies coincide with the fields at which the isolated chain is expected to undergo level crossings. These anomalies are strongly site dependent however. Occasionally they take the typical V-shape of the Kondo resonance of a single impurity split by a magnetic field, but in most cases they are less pronounced if at all.

In this Letter, our goal is to provide a theory of STM that is valid at low temperature and that puts the measurements in the appropriate Kondo context. As we shall see, a Kondo effect is indeed present at each level crossing, but it corresponds to that of an extended impurity of size the length of the chain. The Hilbert space of this extended impurity corresponds to the twofold degenerate ground state of the spin chain at the level crossing. The presence or absence of a Kondo resonance at a given Co site where the STM signal is recorded depends i) on a matrix element encoding the fact that the ground state of the spin chain is addressed at a given Co site, and ii) on the magnitude of the effective Kondo coupling between the extended impurity and the substrate at the considered Co location.

Method. At high temperatures, the differential conductance in the presence of the Kondo coupling can be calculated using perturbation theory. While the results reproduce the gross features of the experimental data, they are limited to a regime above the Kondo temperature, and cannot account for the details of the low temperature 
data of Ref. [11]. For a particle-hole symmetric conduction band, our model can be simulated with the auxiliary field quantum Monte Carlo (QMC) algorithm without encountering the negative sign problem. We have used the finite temperature algorithm ${ }^{13-15}$ of the ALF-project ${ }^{16}$ and followed Refs. [17 and 18] for the implementation of our Kondo model. In the QMC calculation we consider a $20 \times 20$ square lattice with unit lattice constant and hopping matrix element $t$ and consider a linear arrangement of magnetic adatoms at distance $\Delta \boldsymbol{l}=(0,3)$ or $\Delta \boldsymbol{l}=(3,2)$ from each other (up to $L=7$ ). To overcome the finite size effects we included an orbital magnetic field corresponding to a single flux quantum traversing the whole lattice $^{19}$, and a rather large value of the Kondo interaction $J_{k} / t=2$ so as to ensure that the Kondo scale of the single impurity problem remains larger than the finite size level spacing of the conduction electrons. Finally we consider $J_{h} / t=1.8$.

In the case of a single adatom $(L=1)$, the problem reduces to that of a single impurity. The low temperature STM signal observed in Ref. [11] consists of a single peak, the Kondo resonance, consistent with a tunneling process from sample to tip that goes through the localized d-orbital of the Co adatoms. To account for this in the realm of the Kondo model we compute cotunneling processes ${ }^{20-22}$ given by: $A_{\boldsymbol{l}}(\omega)=-\operatorname{Im} G_{l}^{\mathrm{ret}}(\omega)$ with $G_{\boldsymbol{l}}^{\mathrm{ret}}(\omega)=-i \int_{0}^{\infty} d \tau e^{i \omega \tau} \sum_{\sigma}\left\langle\left\{\tilde{d}_{\boldsymbol{l}, \sigma}(\tau), \tilde{d}_{l, \sigma}^{\dagger}(0)\right\}\right\rangle$ and $\tilde{d}_{l, \sigma}^{\dagger}=\hat{c}_{l,-\sigma}^{\dagger} \hat{S}_{l}^{\sigma}+\sigma \hat{c}_{l, \sigma}^{\dagger} \hat{S}_{l}^{z}$. Here $\sigma= \pm$ runs over the two spin polarization and $\hat{S}_{l}^{ \pm}=\hat{S}_{l}^{x} \pm i \hat{S}_{l}^{y}$. This form can be obtained by starting from the single impurity Anderson model and applying the canonical Schrieffer-Wolf transformation (see supplemental material of Ref. [23]) and agrees with the expression given in Ref. [24].

Let us start by showing examples of spectral functions at level crossings obtained from QMC by stochastic analytic continuation ${ }^{25}$. As apparent from Fig. 1a), for a single impurity we observe the characteristic temperature dependence of a Kondo resonance at zero field. Fig. 1
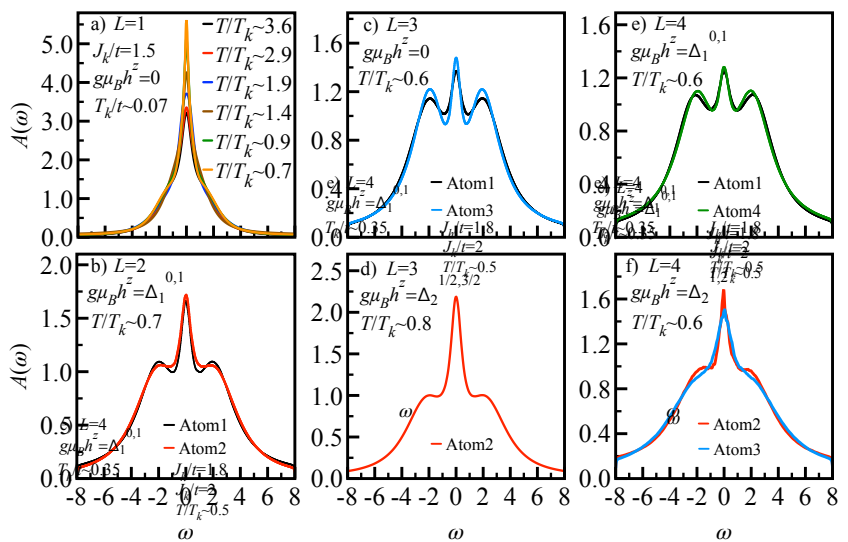

FIG. 1. The spectral function computed using stochastic analytical continuation algorithm ${ }^{25}$ at a given level crossings up to $L=4$. For $L>1$ we choose $J_{h} / t=1.8$ and $J_{k} / t=2$. The corresponding Kondo scale is extracted in Fig. 3. also shows the magnetic field induced Kondo resonances. For the two site chain there is a single level crossing between the singlet and the triplet at $g \mu_{B} h^{z}=\Delta_{1}^{0,1}$. In the generic Kondo problem, time reversal symmetry protects the two-fold degeneracy of the impurity state. Here parity protects the level crossings and a Kondo resonance is apparent on both adatom sites, see Fig. 1b). For the three site chain two level crossings occur before saturation. The ground state is a spin- $1 / 2$ doublet in zero field and resonances are seen on the first and third adatoms, see Fig. 1c). At the second level crossing, the resonance is seen only on the central adatom, see Fig. 1d). For $L=4$, Kondo resonances emerge on outer adatoms at the first level crossing, see Fig.1e), and on the central adatoms for the second level crossing, see Fig. 1.f).

These results have been obtained at temperatures already representative of the low temperature regime, and they reproduce the main features of the experimental results (see Supplemental Material, Ref. [26], Fig. 12). However, to make a quantitative comparison with the experiments, which correspond to much lower temperature, we will concentrate on the zero bias differential conductance measured in the STM experiment ${ }^{10-12}$ as

$$
d I_{l} / d V(V=0)=2 \frac{e^{2}}{\hbar} \int_{-\infty}^{\infty} d \omega\left(-\frac{d f(\omega)}{d \omega}\right) A_{l}(\omega)
$$

where $f(\omega)$ is a Fermi function. In the low temperature limit the above maps onto:

$$
d I_{l} / d V(V=0) \simeq 2 \frac{e^{2}}{\hbar} A_{l}(\omega=0) \simeq 2 \frac{e^{2}}{\pi \hbar} \beta G_{l}(\tau=\beta / 2)
$$

where $G_{\boldsymbol{l}}(\tau)=\sum_{\sigma}\left\langle\tilde{d}_{\boldsymbol{l}, \sigma}(\tau) \tilde{d}_{l, \sigma}^{\dagger}(0)\right\rangle$ is the imaginary time Green function which can be directly computed in the auxiliary field QMC. This approach avoids analytical continuation and our discussion will be based on the field dependence of this quantity. In the zero temperature limit the above equation is exact, and a more precise account of the zero bias differential conductance at finite temperature without using the analytical continuation can be obtained following Refs. [27-29].

The QMC results of the local spectral function at zero frequency for $k_{B} T / t=1 / 30$ are compared to the zero bias conductance reported in Ref. [11] as a function of external magnetic field in Fig. 2. Noticeably, up to four atoms the zero frequency spectral function shows excellent agreement with the corresponding zero bias conductance measured in the experiment. The temperature scales in the QMC and STM are comparable: the data presented in Fig. 2 are computed below $k_{B} T_{k}^{l} / 8 t$, where $T_{k}^{l}$ is an estimate of Kondo temperature from scaling of local spin susceptibility ${ }^{19,30}$ at each level crossings (see below), while the STM data are taken at $330 \mathrm{mK}$ $\sim T_{k}^{C o} / 8\left(k_{B} T / t \sim 1 / 35\right.$ in $\left.\mathrm{QMC}\right)$.

To associate an adatom dependent Kondo temperature to each level crossing, we compute the local transverse susceptibility, $\chi_{l}=\int_{0}^{\beta} d \tau\left\langle\hat{S}_{l}^{+}(\tau) \hat{S}_{l}^{-}(0)+h . c\right\rangle$, where $\hat{S}_{l}^{ \pm}(\tau)=e^{\tau \hat{H}} \hat{S}_{l}^{ \pm} e^{-\tau \hat{H}}$. Interestingly we observe in Fig. 3 


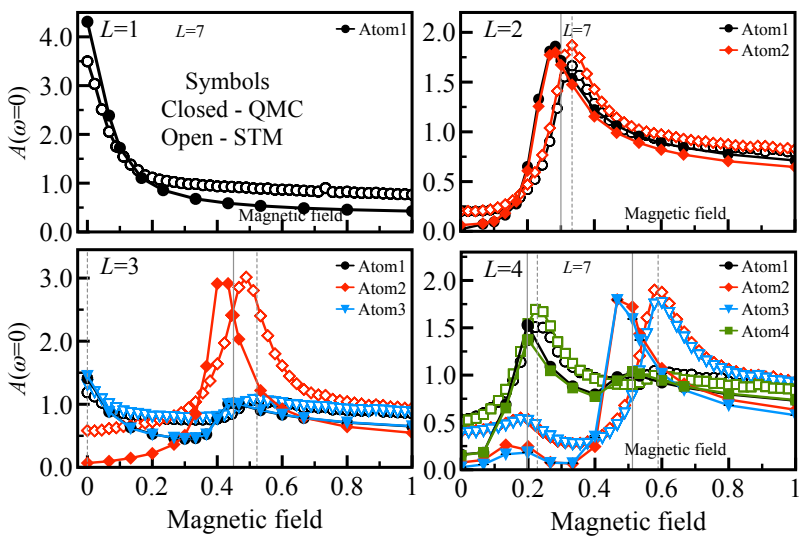

FIG. 2. The $\tilde{d}$-spectral function at $\omega=0$ computed by QMC for a Heisenberg chain in a field together with the zero bias conductance measured in the STM experiment (in atomic units) for an XXZ chain in transverse field ${ }^{11}$. The magnetic field axis is normalised by the maximum values in both cases. The continuous grey vertical lines and the dashed grey lines denote the expected exact positions of the level crossings for a Heisenberg chain and for an XXZ one ${ }^{11}$, respectively.

that when the STM data at an adatom site shows a resonance, the local susceptiblity follows the expected universal behaviour, $T \chi_{l}=f\left(T / T_{k}^{l}\right)$, where $T_{k}^{l}$ corresponds to the adatom and level crossing resolved Kondo temperature. Fig. 4 plots the temperature dependence

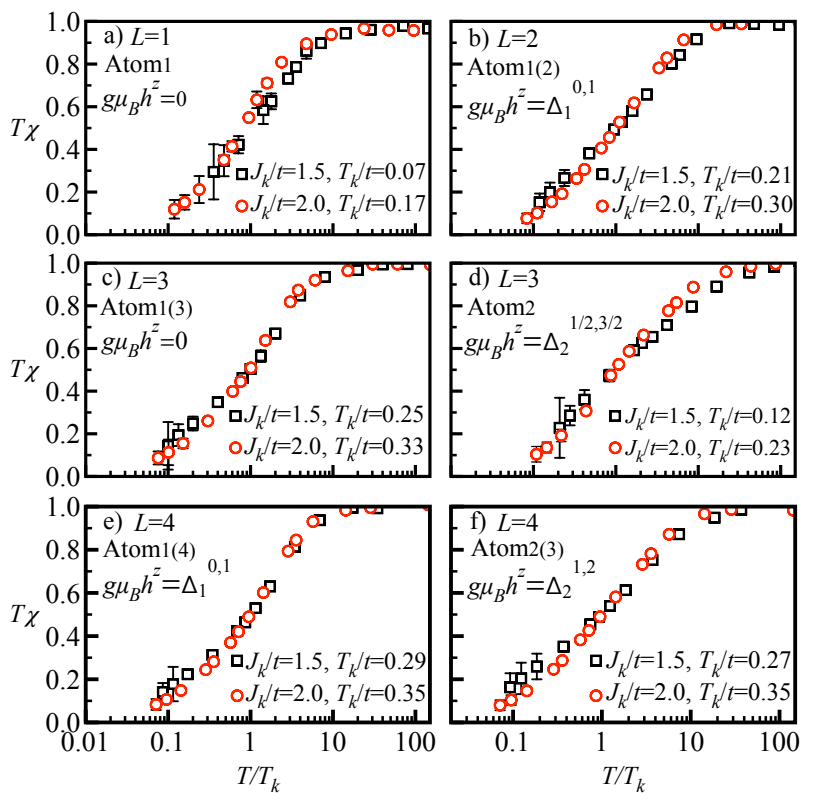

FIG. 3. Local probe transverse susceptibility as a function of temperature computed for $J_{h} / t=1.8$ up to $L=4$ at sites where the level crossing leads to a Kondo resonance. The sites not shown do not follow the typical temperature dependence on Kondo couplings up to $k_{B} T / t \approx 1 / 40$.

of the zero frequency spectral function as estimated by $A_{l}(\omega=0) \simeq \frac{1}{\pi} \beta G_{l}(\tau=\beta / 2)$. For $L=3$ and $L=4$ strong site dependence of the signal emerges below the Kondo temperature. We first concentrate on cases where we observe a dominant resonance. For these cases, the temperature dependence of the zero-bias conductance at the various sites (see Fig. 4) shows logarithmic increase at the Kondo scale upon reducing the temperature. At the other sites no such increase is observed. Quite remarkably, depending on the site, level crossings can show up as a peak, a dip, or a change of slope as a function of field. Accordingly, in the experimental results of Ref. [11], the frequency dependence at a critical field may or may not show a Kondo resonance.
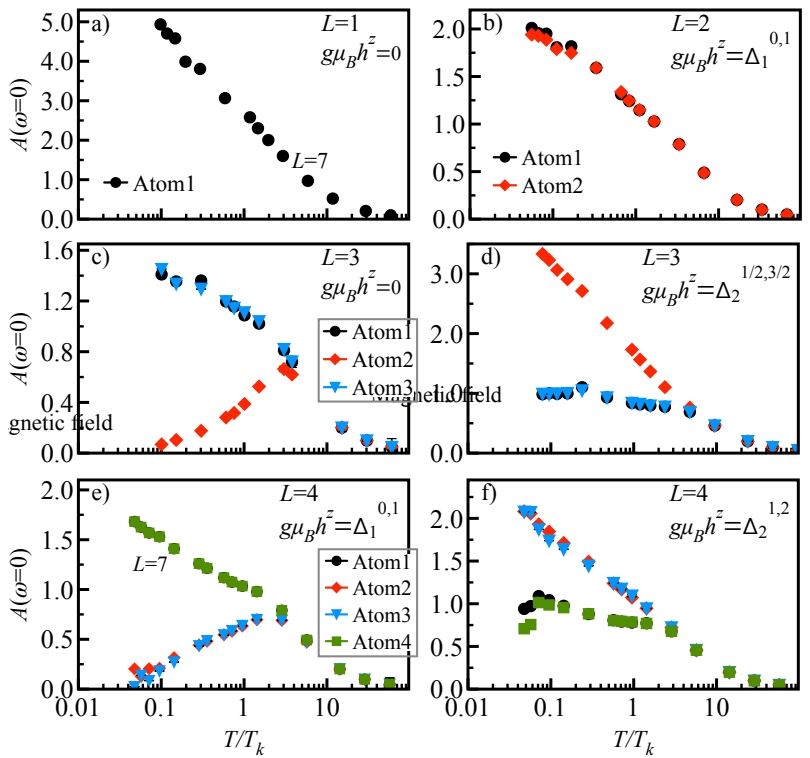

FIG. 4. Exact zero-bias conductance as a function of temperature normalised by the corresponding Kondo scale at each level crossings up to $L=4$.

Effective model. To provide an effective model for a given level crossing, $p$, we project the Hamiltonian on the two fold degenerate Hilbert space of the level crossing. Clearly such a strategy is valid only if the gap separating the next excited states of the spin chain is large compared to the effective Kondo scale. For our SU(2) model, this approximation will necessarily fail in the large $L$ limit, but as we will see below it provides an accurate account of the QMC data for small $L$. Let $\left\{\left|m_{\mathbf{1}}\right\rangle=\left|\mathbf{S}_{m_{1}} \mathbf{S}_{m_{1}}^{z}\right\rangle_{p},\left|m_{\mathbf{2}}\right\rangle=\left|\mathbf{S}_{m_{\mathbf{2}}} \mathbf{S}_{m_{\mathbf{2}}}^{z}\right\rangle_{p}\right\}$ be the eigenstate of the Heisenberg chain with energies $e_{m_{1}, p}, e_{m_{2}, p}$ that span the Hilbert space of the level crossing and $\hat{P}$ the projector onto this space. Defining a vector of Pauli spin matrices $\tau$ that act on this Hilbert space, the projected Hamiltonian, up to a constant, reads:

$$
\begin{aligned}
& \hat{H}_{p}^{e f f}=-t \sum_{\langle\boldsymbol{i}, \boldsymbol{j}\rangle, \sigma}\left(\hat{c}_{\boldsymbol{i}, \sigma}^{\dagger} \hat{c}_{\boldsymbol{j}, \sigma}+h . c\right)+J_{k} \sum_{\boldsymbol{l}}\left\{j _ { \boldsymbol { l } , p } ^ { \perp } \left(\hat{S}_{\boldsymbol{l}}^{x, c} \hat{\tau}^{x}+\right.\right. \\
& \left.\left.\left.\hat{S}_{\boldsymbol{l}}^{y, c} \hat{\tau}^{y}\right)+j_{\boldsymbol{l}, p}^{z} \hat{S}_{\boldsymbol{l}}^{z, c} \hat{\tau}^{z}+\mu_{\boldsymbol{l}, p} S_{\boldsymbol{l}}^{z, c}+\left(\Delta_{p}^{m_{\mathbf{1}}, m_{\mathbf{2}}}-g \mu_{B} h^{\mathbf{z}}\right) \hat{n}\right\} 4\right)
\end{aligned}
$$

The energy difference between the two local states is 


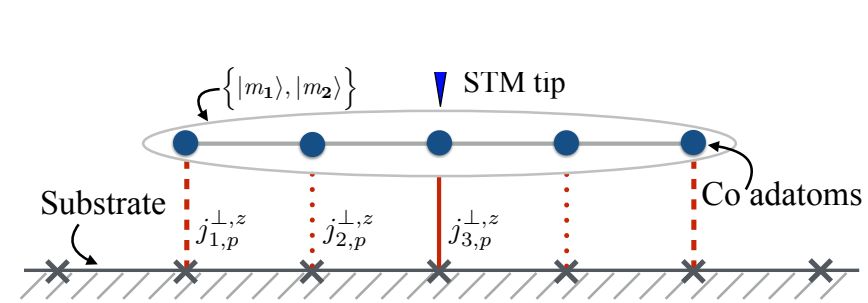

FIG. 5. A chain of Co adatoms on a substrate implement, at a level crossing, the Kondo model of an extended impurity.

TABLE I. Effective couplings $j_{l\left(\boldsymbol{l}^{\prime}\right), p}^{\perp, z}$ at the level $\operatorname{crossings}(p=$ $1, \cdots, \frac{L}{2}\left(\frac{L+1}{2}\right)$ for a fixed even(odd) $L$.)

\begin{tabular}{l|l}
\hline \hline$L=2$ & $j_{1(2), 1}^{\perp}=\frac{1}{2 \sqrt{2}}, \quad j_{1(2), 1}^{z}=\frac{1}{4}$ \\
$L=3$ & $j_{1(3), 1}^{\perp}=\frac{1}{3}, \quad j_{2,1}^{\perp}=\frac{1}{6}, \quad j_{1(3), 1}^{z}=\frac{1}{3}, \quad j_{2,1}^{z}=\frac{1}{6}$ \\
$L=3$ & $j_{1(3), 2}^{\perp}=\frac{1}{2 \sqrt{6}}, \quad j_{2,2}^{\perp}=\frac{1}{\sqrt{6}}, \quad j_{1(3), 2}^{z}=\frac{1}{12}, \quad j_{2,2}^{z}=\frac{1}{3}$ \\
$L=4$ & $j_{1(4), 1}^{\perp}=\frac{1}{4 \sqrt{6} \sqrt{2+\sqrt{2}}}\left[\frac{1+\sqrt{2}}{1+\sqrt{3}}+1+\sqrt{1+\frac{\sqrt{3}}{2}}(1+\sqrt{2})\right]$ \\
& $j_{2(3), 1}^{\perp}=\frac{1}{4 \sqrt{6} \sqrt{2+\sqrt{2}}}\left[\frac{1}{1+\sqrt{3}}+(1+\sqrt{2})+\sqrt{1+\frac{\sqrt{3}}{2}}\right]$ \\
$L=4$ & $j_{1(4), 1}^{z}=\frac{1}{16}(2+\sqrt{2}), \quad j_{2(3), 1}^{z}=\frac{1}{16}(2-\sqrt{2})$ \\
$j_{1(4), 2}^{\perp}=\frac{1}{4 \sqrt{2+\sqrt{2}}}, \quad j_{2(3), 2}^{\perp}=\frac{1+\sqrt{2}}{4 \sqrt{2+\sqrt{2}}}$ \\
$j_{1(4), 2}^{z}=\frac{1}{16}(2-\sqrt{2}), \quad j_{2(3), 2}^{z}=\frac{1}{16}(2+\sqrt{2})$ \\
\hline
\end{tabular}

given by $\Delta_{p}^{m_{1}, m_{2}}=e_{m_{\mathbf{2}}, p}-e_{m_{\mathbf{1}}, p}, \hat{n}=\frac{1}{2}\left(\hat{\mathbf{1}}+\hat{\tau}^{z}\right)$, and the site dependent effective couplings and the effective magnetic field are defined as; $j_{\boldsymbol{l}, p}^{\perp}=\left\langle m_{\mathbf{2}}\left|\hat{S}_{\boldsymbol{l}}^{x, y}\right| m_{\mathbf{1}}\right\rangle, 2 j_{\boldsymbol{l}, p}^{z}=$ $-\left\langle m_{\mathbf{1}}\left|\hat{S}_{\boldsymbol{l}}^{z}\right| m_{\mathbf{1}}\right\rangle+\left\langle m_{\mathbf{2}}\left|\hat{S}_{\boldsymbol{l}}^{z}\right| m_{\mathbf{2}}\right\rangle$ and $2 \mu_{\boldsymbol{l}, p}=\left\langle m_{\mathbf{1}}\left|\hat{S}_{\boldsymbol{l}}^{z}\right| m_{\mathbf{1}}\right\rangle+$ $\left\langle m_{\mathbf{2}}\left|\hat{S}_{\boldsymbol{l}}^{z}\right| m_{\mathbf{2}}\right\rangle$.

This model can be interpreted as the Kondo model of an extended impurity that is coupled to the conduction electrons at $L$ points (see Fig. 5 ). The projection does not affect the $\mathrm{U}(1)$ spin symmetry of the model, but as shown in Table I it yields a strong site dependence of the effective couplings $j_{l, p}^{\perp, z}$. To compute the co-tunneling within the effective model, we still have to project the spin operator onto the level-crossing Hilbert space $\hat{P} \tilde{d}_{\boldsymbol{l}, \sigma}^{\dagger} \hat{P}=\hat{c}_{\boldsymbol{l},-\sigma}^{\dagger} \hat{P} \hat{S}_{\boldsymbol{l}}^{\sigma} \hat{P}+\sigma \hat{c}_{\boldsymbol{l}, \sigma}^{\dagger} \hat{P} S_{\boldsymbol{l}}^{z} \hat{P}$ so that it acquires a site dependence when written in terms of $\tau$ operators. This reflects the fact that in the experiment the extended states $\left|m_{\mathbf{1}}\right\rangle$ and $\left|m_{\mathbf{2}}\right\rangle$ are addressed via manipulation of one of the constituent Co d-spins. A detailed numerical analysis of the effective model along these lines is left for future studies. However, it is already clear that it provides a qualitative interpretation of the data in terms of a projection induced hierarchy of Kondo scales (see Ref. [26], Table IV). Consider for instance the four-site chain at the first, $p=1$, level crossing for which $j_{1(4), 1}^{z} / j_{2(3), 1}^{z}=5.83$ and $j_{1(4), 1}^{\perp} / j_{2(3), 1}^{\perp}=1.25$. Thereby, the Kondo singlet will be predominantly formed by an entangled state of the degenerate two level system, $\left\{\left|m_{\mathbf{1}}\right\rangle,\left|m_{\mathbf{2}}\right\rangle\right\}$ and a symmetric combination of the conduction electron spins on sites one and four (see Ref. [26],

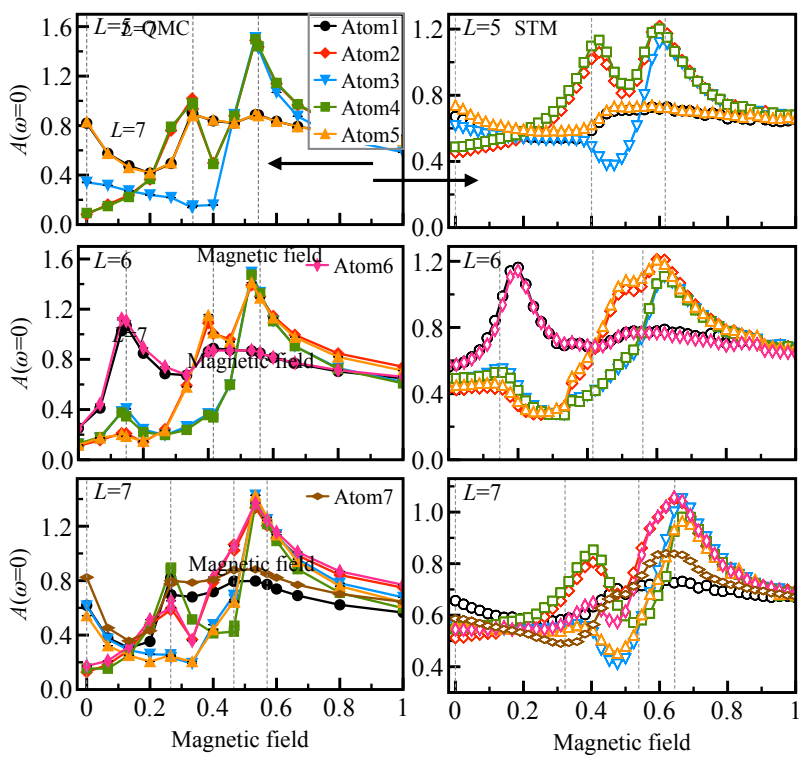

FIG. 6. QMC results for five, six and seven atom spin-chains together with the STM data around $V \sim 0.6 \mathrm{mV}^{11}$ as a function of magnetic field (normalised by the maximum values).

Section B.). This provides an understanding of the observed Kondo like temperature dependence of the zerobias conductance at sites one and four.

In Fig. 6 we consider chains up to seven spins. Here, an accurate comparison of the zero bias conductance is hard due to the asymmetric line shape of the Kondo resonances that arise in the experiment due to the potential scattering between tip and sample ${ }^{20-22,31}$, but a reasonable agreement can be achieved slightly away from the zero bias around $V \sim 0.4-0.8 \mathrm{mV}$ as shown in Fig. 6 . As the chain size grows, the spectrum will collapse, and our understanding in terms of the projection onto the two-fold level crossing Hilbert space fails. In fact in this limit we expect a crossover to Kondo lattice behavior characterized at the mean field level by hybridized heavy and light bands in a magnetic field (see Ref. [26], Section $\mathrm{C}$ ). For an infinite Heisenberg chain and within this approximation, we expect the conductance to reflect the local spinon density of states.

To summarize, we have shown that the STM measurements on Co adatom chains agree remarkably well with QMC simulations of a model of spin-1/2 chains in a field coupled to a conducting substrate via local Kondo couplings. To interpret the strong site dependence of the signal, we have performed a projection onto the symmetry protected level crossing Hilbert space of the spin chain that leads to the notion of an extended impurity with site dependent Kondo couplings. Consequently, screening happens via the dominant channel such that site and level crossing dependent Kondo resonances are observed in the STM co-tunneling conductance as well as in the Monte Carlo simulations. As a function of chain length, the above construction will progressively fail and we expect a crossover to Kondo lattice physics, in which the 
STM signal will pick up the two spinon continuum of the spin chain. Further work at understanding the details of the extended impurity Kondo model, as well as the crossover to the lattice limit is presently under progress.

\section{ACKNOWLEDGMENTS}

We thank S. Otte, M. Raczkowski, and M. Ternes for very useful discussions and S. Otte and M. Ternes for providing the STM data. FFA thanks the DFG collaborative research centre SFB1170 To CoTronics (project C01) for financial support as well as the Würzburg-Dresden Clus- ter of Excellence on Complexity and Topology in Quantum Matter - ct.qmat (EXC 2147, project-id 39085490). The authors gratefully acknowledge the Gauss Centre for Supercomputing e.V. (www.gauss-centre.eu) for funding this project by providing computing time on the GCS Supercomputer SUPERMUC-NG at Leibniz Supercomputing Centre (www.lrz.de). FM and BD acknowledge the Swiss National Science Foundation $t$ and its SINERGIA network Mott physics beyond the Heisenberg model for financial support. This work was also supported by EPFL through the use of the facilities of its Scientific IT and Application Support Center. *danu.bimla@epfl.ch

$\dagger$ assaad@physik.uni-wuerzburg.de

‡ frederic.mila@epfl.ch

1 A. C. Hewson, The Kondo Problem to Heavy Fermions, Cambridge Studies in Magnetism (Cambridge University Press, 1993).

2 J. Kondo, Progress of Theoretical Physics 32, 37 (1964).

${ }^{3}$ K. G. Wilson, Rev. Mod. Phys. 47, 773 (1975).

4 V. Madhavan, W. Chen, T. Jamneala, M. F. Crommie, and N. S. Wingreen, Science 280, 567 (1998).

5 S. M. Cronenwett, T. H. Oosterkamp, and L. P. Kouwenhoven, Science 281, 540 (1998).

6 J. Park, A. N. Pasupathy, J. I. Goldsmith, C. Chang, Y. Yaish, J. R. Petta, M. Rinkoski, J. P. Sethna, H. D. Abruna, P. L. McEuen, and D. C. Ralph, Nature 417 (2002).

7 P. Jarillo-Herrero, J. Kong, H. S. J. van der Zant, C. Dekker, L. P. Kouwenhoven, and S. De Franceschi, Nature 434 (2005).

8 P. Wahl, L. Diekhöner, G. Wittich, L. Vitali, M. A. Schneider, and K. Kern, Phys. Rev. Lett. 95, 166601 (2005).

9 N. Néel, R. Berndt, J. Kröger, T. O. Wehling, A. I. Lichtenstein, and M. I. Katsnelson, Phys. Rev. Lett. 107, 106804 (2011).

10 A. Spinelli, M. Gerrits, R. Toskovic, B. Bryant, M. Ternes, and A. F. Otte, Nature Communications 6 (2015).

11 R. Toskovic, R. van den Berg, A. Spinelli, I. S. Eliens, B. van den Toorn, B. Bryant, J. S. Caux, and A. F. Otte, Nature Physics 12 (2016).

12 A. F. Otte, M. Ternes, K. von Bergmann, S. Loth, H. Brune, C. P. Lutz, C. F. Hirjibehedin, and A. J. Heinrich, Nature Physics 4, 847 EP (2008).

13 R. Blankenbecler, D. J. Scalapino, and R. L. Sugar, Phys. Rev. D 24, 2278 (1981).

14 S. R. White, D. J. Scalapino, R. L. Sugar, E. Y. Loh, J. E. Gubernatis, and R. T. Scalettar, Phys. Rev. B 40, 506 (1989).

15 F. Assaad and H. Evertz, in Computational Many-Particle Physics, Lecture Notes in Physics, Vol. 739, edited by H. Fehske, R. Schneider, and A. Weiße (Springer, Berlin Heidelberg, 2008) pp. 277-356.

16 M. Bercx, F. Goth, J. S. Hofmann, and F. F. Assaad, SciPost Phys. 3, 013 (2017).

17 F. F. Assaad, Phys. Rev. Lett. 83, 796 (1999).

18 T. Sato, F. F. Assaad, and T. Grover, Phys. Rev. Lett.
120, 107201 (2018).

19 F. F. Assaad, Phys. Rev. B 65, 115104 (2002).

20 J. Figgins and D. K. Morr, Phys. Rev. Lett. 104, 187202 (2010).

21 M. Ternes, New Journal of Physics 17, 063016 (2015).

22 D. K. Morr, Reports on Progress in Physics 80, 014502 (2017).

23 M. Raczkowski and F. F. Assaad, Phys. Rev. Lett. 122, 097203 (2019).

24 T. A. Costi, Phys. Rev. Lett. 85, 1504 (2000).

${ }^{25}$ K. S. D. Beach, arxiv:0403055 (2004).

${ }^{26}$ For details on the level crossings, the effective model, the Kondo scales, the mean-field Hamiltonian of an infinite chain, and $d I / d V$ spectrum of STM experiment, see Supplemental Material.

27 D. J. Luitz, F. F. Assaad, T. Novotný, C. Karrasch, and V. Meden, Phys. Rev. Lett. 108, 227001 (2012).

${ }^{28}$ C. Karrasch, V. Meden, and K. Schönhammer, Phys. Rev. B 82, 125114 (2010).

29 H. Monien, Math. Comp. 79, 857 (2010).

30 J. E. Hirsch and R. M. Fye, Phys. Rev. Lett. 56, 2521 (1986)

31 K. von Bergmann, M. Ternes, S. Loth, C. P. Lutz, and A. J. Heinrich, Phys. Rev. Lett. 114, 076601 (2015).

32 P. W. Anderson, G. Yuval, and D. R. Hamann, Phys. Rev. B 1, 4464 (1970).

${ }^{33}$ P. W. Anderson, Journal of Physics C: Solid State Physics 3, 2436 (1970).

${ }^{34}$ K. Yosida, Theory of Magnetism, Springer series in solid sciences (Springer-Verlag, 1991).

35 C. Romeike, M. R. Wegewijs, W. Hofstetter, and H. Schoeller, Phys. Rev. Lett. 96, 196601 (2006).

${ }^{36}$ R. Žitko, R. Peters, and T. Pruschke, Phys. Rev. B 78, 224404 (2008). 


\section{Supplemental Material for: Exploring the Kondo effect of an extended impurity with chains of Co adatoms in a magnetic field}

\section{A. Effective models at level crossings}

To derive the effective model at level crossing $p$ (see Fig. 9 and Fig. 10) we project the Hamiltonian of Eq. (1) onto the Hilbert space spanned by the states $\left\{\left|m_{\mathbf{1}}\right\rangle,\left|m_{\mathbf{2}}\right\rangle\right\}$ of the level crossing of a finite Heisenberg chain at a given critical magnetic field.

$$
\begin{array}{r}
\hat{H}_{p}^{e f f}=-t \sum_{\langle\boldsymbol{i}, \boldsymbol{j}\rangle, \sigma}\left(\hat{c}_{\boldsymbol{i}, \sigma}^{\dagger} \hat{c}_{\boldsymbol{j}, \sigma}+h . c\right)+J_{k} \sum_{\boldsymbol{l}=1}^{L} \sum_{m m^{\prime}=m_{\mathbf{1}}, m_{\mathbf{2}}} \mathbf{S}_{\boldsymbol{l}}^{c} \cdot\left|m^{\prime}\right\rangle\left\langle m^{\prime}\left|\mathbf{S}_{\boldsymbol{l}}\right| m\right\rangle\langle m| \\
+\left(e_{m_{\mathbf{1}}, p}-\mathbf{S}_{m_{\mathbf{1}}, p}^{z} h^{z}\right)\left|m_{\mathbf{1}}\right\rangle\left\langle m_{\mathbf{1}}\left|+\left(e_{m_{\mathbf{2}}, p}-\mathbf{S}_{m_{\mathbf{2}}, p}^{z} h^{z}\right)\right| m_{\mathbf{2}}\right\rangle\left\langle m_{\mathbf{2}}\right| .
\end{array}
$$

The states are explicitly given in Table II. We further introduce pseudo spin-1/2 operators; $\hat{\tau}^{x}=\left|m_{\mathbf{2}}\right\rangle\left\langle m_{\mathbf{1}}|+| m_{\mathbf{1}}\right\rangle\left\langle m_{\mathbf{2}}\right|$,

TABLE II. The subspace $\left\{\left|m_{\mathbf{1}}\right\rangle=\left|\mathbf{S}_{m_{\mathbf{1}}} \mathbf{S}_{m_{\mathbf{1}}}^{z}\right\rangle_{p},\left|m_{\mathbf{2}}\right\rangle=\left|\mathbf{S}_{m_{\mathbf{2}}} \mathbf{S}_{m_{\mathbf{2}}}^{z}\right\rangle_{p}\right\}$ at a level crossing $p$ of a finite Heisenberg chain $\left(J_{h} \sum_{l=1}^{L-1} \hat{\boldsymbol{S}}_{l}\right.$. $\hat{\boldsymbol{S}}_{l+1}$ ) and corresponding eigenenergies, lowest excitation gap, and effective magnetic field.

\begin{tabular}{l|l}
\hline \hline$L=2$ & $|0\rangle=\frac{1}{\sqrt{2}}(|\uparrow \downarrow\rangle-|\downarrow \uparrow\rangle), \quad|1\rangle=|\uparrow \uparrow\rangle$ \\
& $e_{0,1}=-\frac{3}{4} J_{h}, \quad e_{1,1}=\frac{J_{h}}{4}, \quad \Delta_{1}^{0,1}=J_{h}, \quad \mu_{1(2), 1}=\frac{1}{4}$ \\
& $\left.\left.\left|\frac{1}{2}\right\rangle=\frac{1}{\sqrt{6}}(|\downarrow \uparrow \uparrow\rangle-2|\uparrow \downarrow \uparrow\rangle+\uparrow \uparrow \downarrow\rangle\right), \quad\left|-\frac{1}{2}\right\rangle=\frac{1}{\sqrt{6}}(|\downarrow \downarrow \uparrow\rangle-2|\downarrow \uparrow \downarrow\rangle+\uparrow \downarrow \downarrow\rangle\right)$ \\
& $e_{\frac{1}{2}, 1}=-J_{h}, \quad e_{-\frac{1}{2}, 1}=-J_{h}, \quad \Delta_{1}^{-\frac{1}{2}, \frac{1}{2}}=0, \quad \mu_{1(3), 1}=0, \quad \mu_{2,1}=0$ \\
& $\left|\frac{1}{2}\right\rangle=\frac{1}{\sqrt{6}}(|\downarrow \uparrow \uparrow\rangle-2|\uparrow \downarrow \uparrow\rangle+|\uparrow \uparrow \downarrow\rangle), \quad\left|\frac{3}{2}\right\rangle=|\uparrow \uparrow \uparrow\rangle$ \\
& $e_{\frac{1}{2}, 2}=-J_{h}, \quad e_{\frac{3}{2}, 2}=\frac{J_{h}}{2}, \quad \Delta_{2}^{\frac{1}{2}, \frac{3}{2}}=\frac{3}{2} J_{h}, \quad \mu_{1(3), 2}=\frac{5}{12}, \quad \mu_{2,2}=\frac{1}{6}$ \\
& $|0\rangle=\frac{1}{\sqrt{6}}\left[\frac{1}{(1+\sqrt{3})}(|\downarrow \downarrow \uparrow \uparrow\rangle+|\uparrow \uparrow \downarrow \downarrow\rangle)+|\downarrow \uparrow \uparrow \downarrow\rangle+|\uparrow \downarrow \downarrow \uparrow\rangle-\sqrt{1+\frac{\sqrt{3}}{2}}(|\downarrow \uparrow \downarrow \uparrow\rangle+|\uparrow \downarrow \uparrow \downarrow\rangle)\right]$ \\
& $|1\rangle=\frac{1}{2 \sqrt{2+\sqrt{2}}}[-|\downarrow \uparrow \uparrow \uparrow\rangle+|\uparrow \uparrow \uparrow \downarrow+(1+\sqrt{2})(|\uparrow \downarrow \uparrow \uparrow\rangle-|\uparrow \uparrow \downarrow \uparrow\rangle)]$ \\
& $e_{0,1}=\frac{J_{h}}{4}(-3-2 \sqrt{3}), \quad e_{1,1}=\frac{J_{h}}{4}(-1-2 \sqrt{2}), \quad \Delta_{1}^{0,1}=\frac{J_{h}}{2}(1-\sqrt{2}+\sqrt{3}), \quad \mu_{1(4), 1}=\frac{(2+\sqrt{2})}{16}, \quad \mu_{2(3), 1}=\frac{(2-\sqrt{2})}{16}$ \\
& $|1\rangle=\frac{1}{2 \sqrt{2+\sqrt{2}}}[-|\downarrow \uparrow \uparrow \uparrow\rangle+|\uparrow \uparrow \uparrow \downarrow+(1+\sqrt{2})(|\uparrow \downarrow \uparrow \uparrow\rangle-|\uparrow \uparrow \downarrow \uparrow\rangle)], \quad|2\rangle=|\uparrow \uparrow \uparrow \uparrow\rangle$ \\
& $e_{1,2}=\frac{J_{h}}{4}(-1-2 \sqrt{2}), \quad e_{2,2}=\frac{3 J_{h}}{4}, \quad \Delta_{2}^{1,2}=J_{h}\left(1+\frac{1}{\sqrt{2}}\right), \quad \mu_{1(4), 2}=\frac{(6+\sqrt{2})}{16}, \quad \mu_{2(3), 2}=\frac{(6-\sqrt{2})}{16}$
\end{tabular}

$\hat{\tau}^{y}=-i\left(\left|m_{\mathbf{2}}\right\rangle\left\langle m_{\mathbf{1}}|-| m_{\mathbf{1}}\right\rangle\left\langle m_{\mathbf{2}}\right|\right)$ and $\hat{\tau}^{z}=\left|m_{\mathbf{2}}\right\rangle\left\langle m_{\mathbf{2}}|-| m_{\mathbf{1}}\right\rangle\left\langle m_{\mathbf{1}}\right|$ and impose the constraint $\hat{\mathbf{1}}=\left|m_{\mathbf{1}}\right\rangle\left\langle m_{\mathbf{1}}|+| m_{\mathbf{2}}\right\rangle\left\langle m_{\mathbf{2}}\right|$. In terms of the $\boldsymbol{\tau}$ operators Eq. (5) takes the form given in Eq. (4) with a set of site dependent effective couplings given in Table I. The other effective parameters of Eq. (4) are given in Table II up to L=4. When calculating the local matrix elements, we obtain an alternative $\pm \operatorname{sign}$ of $j_{l, p}^{\perp}$ which does not affect Kondo physics ${ }^{32,33}$. For simplicity we omitted this sign $(-1)^{l}$ in Table I of the main text.

Noticeably, for a Heisenberg chain $\left(J_{h} \sum_{l=1}^{L-1} \hat{\boldsymbol{S}}_{\boldsymbol{l}} \cdot \hat{\boldsymbol{S}}_{\boldsymbol{l + 1}}\right)$ the effective couplings $\left(j_{\boldsymbol{l}\left(\boldsymbol{l}^{\prime}\right), p}^{\perp, z}\right)$ are independent of $J_{h}$ (see Table I). However, for an XXZ chain $\left(J_{x y} \sum_{\boldsymbol{l}=1}^{L-1}\left(\hat{S}^{x}{ }_{\boldsymbol{l}} \hat{S}^{x} \boldsymbol{l}+1+\hat{S^{y}}{ }_{\boldsymbol{l}} \hat{S}^{y} \boldsymbol{l}+1\right)+J_{z z} \sum_{\boldsymbol{l}=1}^{L-1} \hat{S}_{\boldsymbol{l}} \hat{S}^{z} \boldsymbol{l + 1}\right)$ the ratio of the exchange parameters $J_{z z} / J_{x y}$ appears in the expression of the effective couplings. Since they do not have a simple form, we choose to plot them as a function of $J_{z z} / J_{x y}$ in Fig.7 and Fig.8 for chains of three and four atoms respectively. As for the Heisenberg chain $\left(J_{z z} / J_{x y}=1\right)$, there is always a strong site dependence of the effective couplings when varying $J_{z z} / J_{x y}=0, \cdots, 1$. Hence, the magnetic field induced level crossings in finite XXZ and Heisenberg chains is expected to show a similar site dependent Kondo physics. 

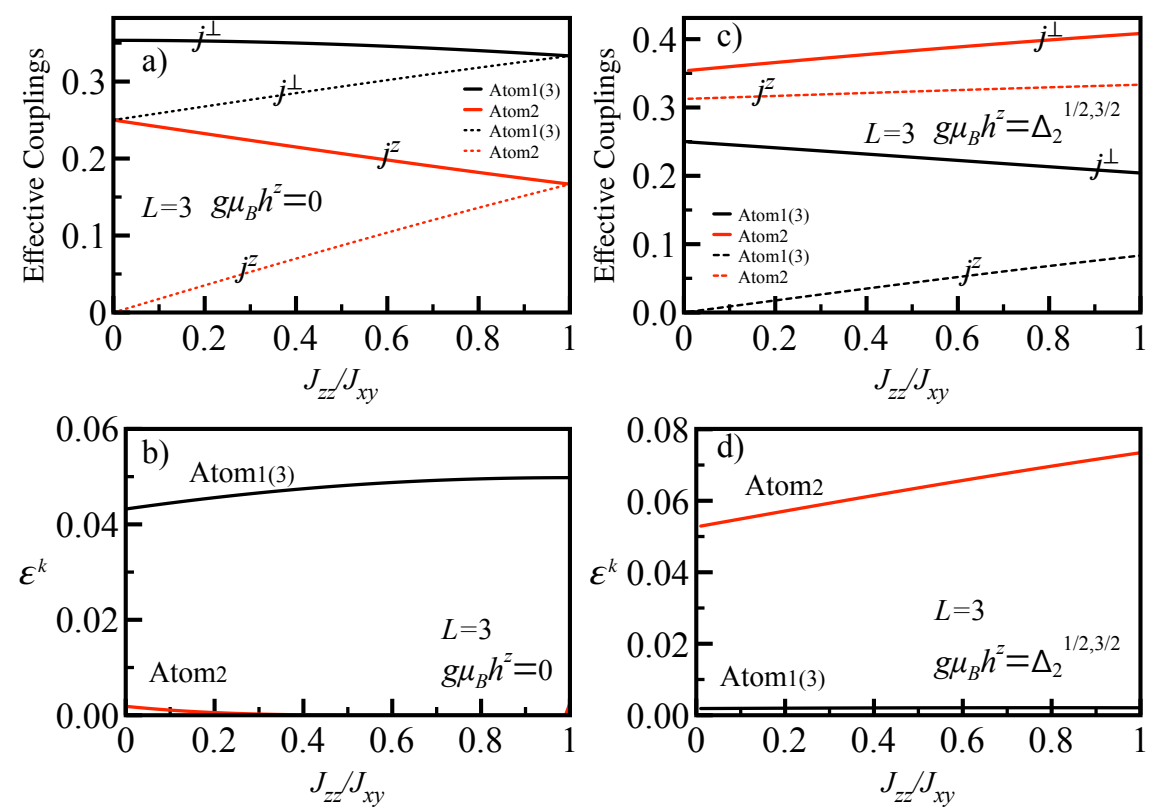

FIG. 7. Top: Site dependent effective couplings as a function of $J_{z z} / J_{x y}$ at level crossings for $L=3$. Bottom: Corresponding effective Kondo scale as a function of $J_{z z} / J_{x y}$. Here, the effective Kondo scale is estimated as; $\epsilon_{l\left(l^{\prime}\right), p}^{k} \sim e^{-\frac{1}{j_{l, p}}}$ with $\frac{1}{j_{l, p}}=$ $\frac{1}{\sqrt{\left(j_{l, p}^{\perp}\right)^{2}-\left(j_{l, p}^{z}\right)^{2}}} \times \tan ^{-1}\left(\frac{\sqrt{\left(j_{l, p}^{\perp}\right)^{2}-\left(j_{l, p}^{z}\right)^{2}}}{j_{l, p}^{z}}\right)$ (see Section B.).
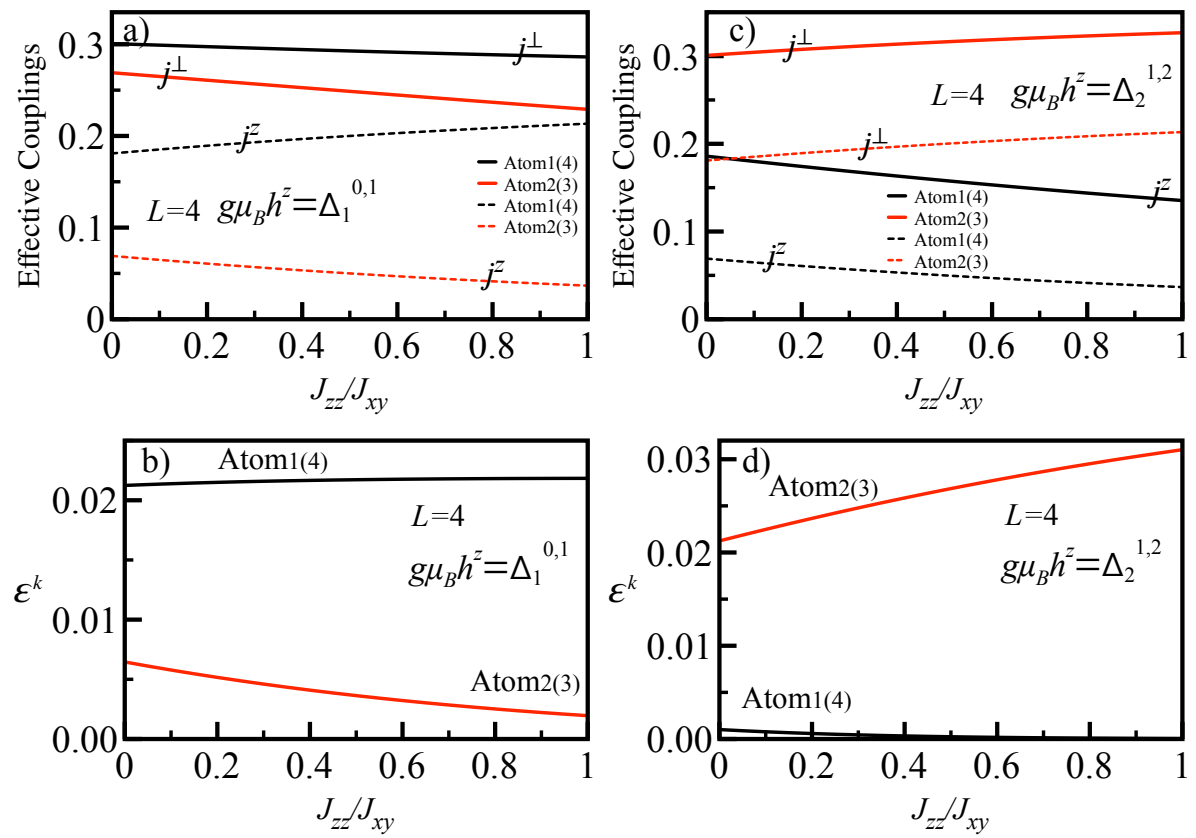

FIG. 8. Top: Site dependent effective couplings as a function of $J_{z z} / J_{x y}$ at level crossings for $L=4$. Bottom: Corresponding effective Kondo scale $\left(\epsilon_{l\left(l^{\prime}\right), p}^{k} \sim e^{-\frac{1}{j_{l, p}}}\right)$ as a function of $J_{z z} / J_{x y}$. 

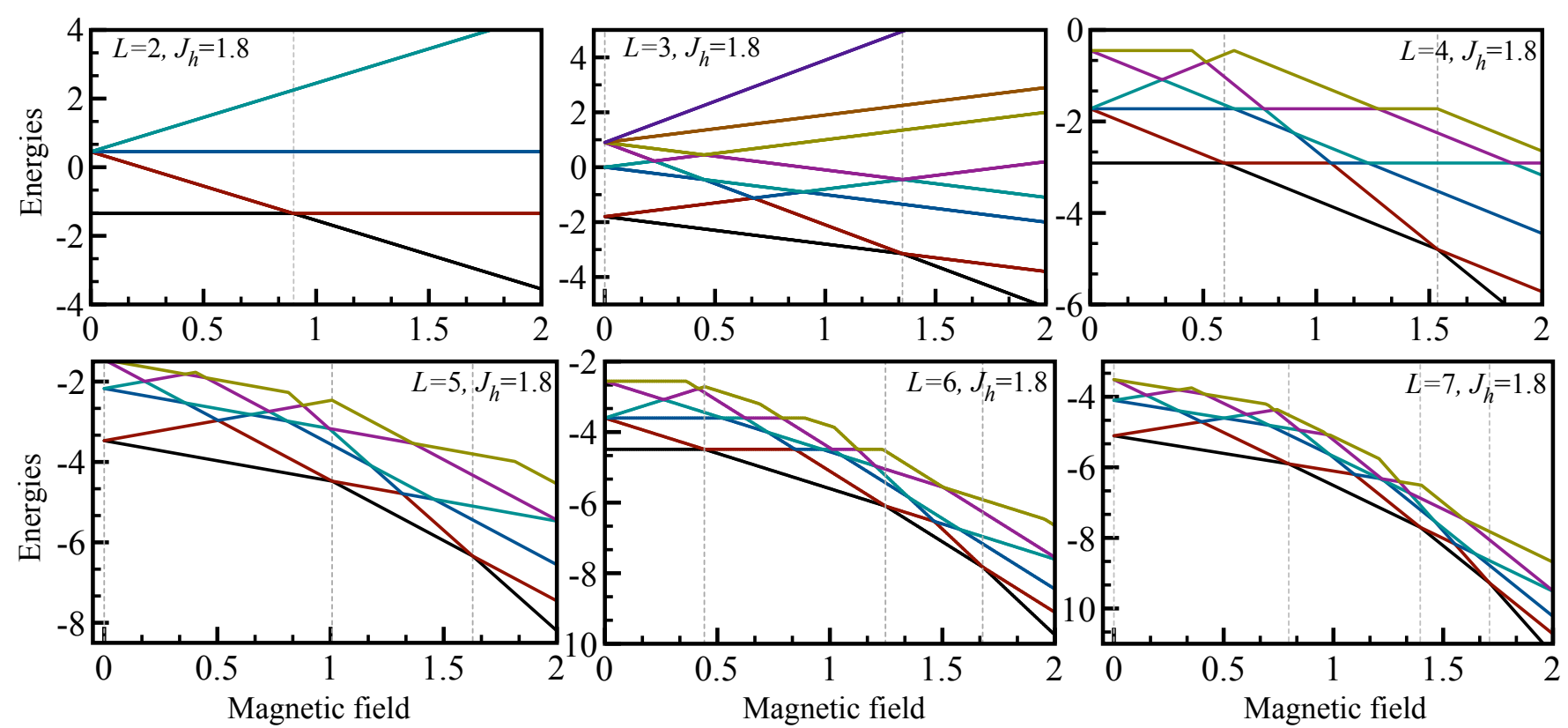

FIG. 9. Lowest eigen energies as a function of magnetic field (in $z$-direction in units of $g \mu_{B}$ ) for a spin- $1 / 2$ Heisenberg chain.
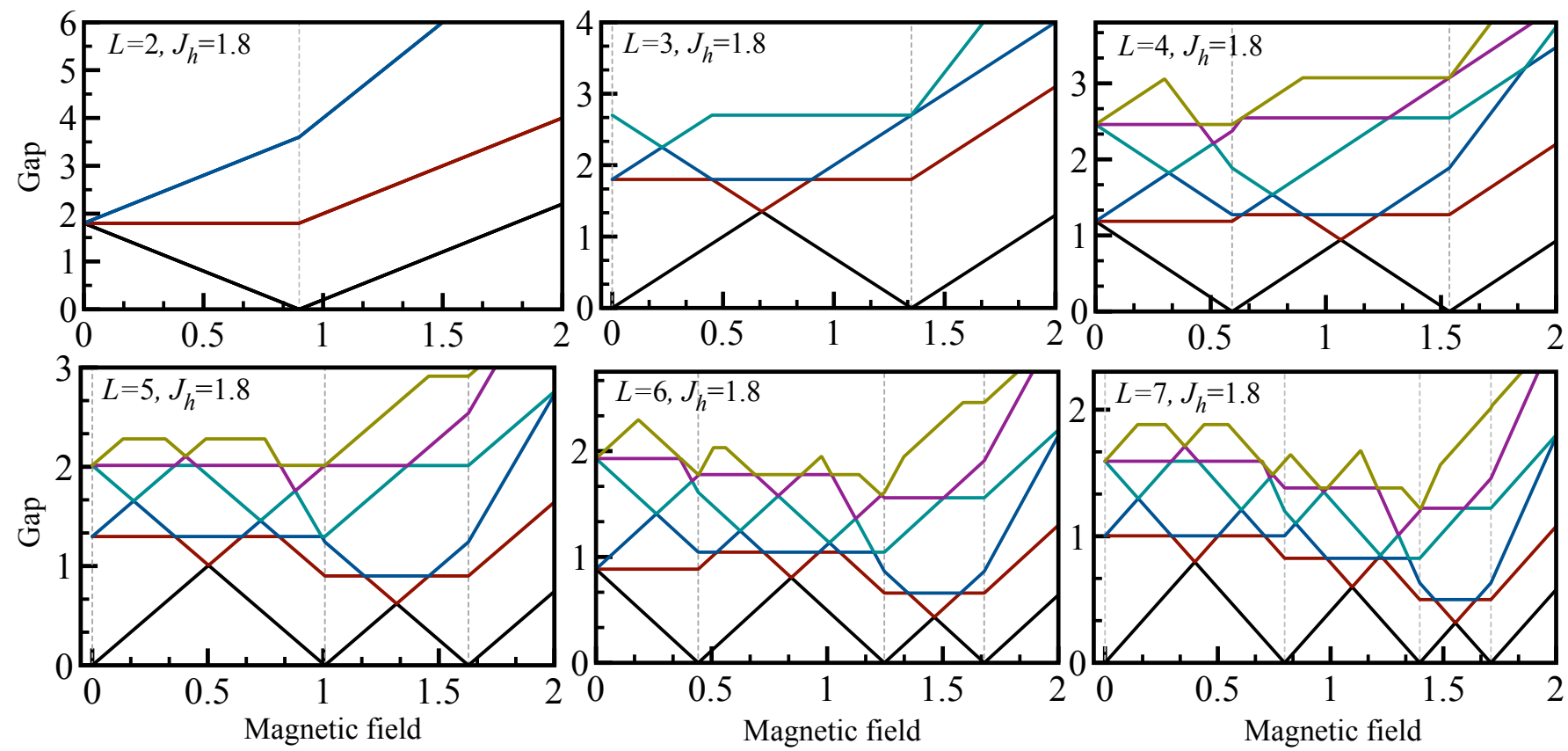

FIG. 10. Lowest energy excitations as a function of magnetic field (in $z$-direction in units of $g \mu_{B}$ ) for a spin-1/2 Heisenberg chain.

B. Effective Kondo Scale at level crossings (Anderson Poor man scaling approach)

Starting from the effective Kondo Hamiltonian for a chain of two atoms at singlet-triplet level crossing, which reads

$$
\begin{aligned}
\hat{H}_{1}^{e f f}= & e_{0,1}-t \sum_{\langle i, j\rangle, \sigma}\left(\hat{c}_{i, \sigma}^{\dagger} \hat{c}_{j, \sigma}+h . c\right)+J_{k}\left\{j^{\perp}\left[\left(\hat{S}_{l_{1}}^{x, c}+\hat{S}_{l_{2}}^{x, c}\right) \hat{\tau}^{x}+\left(\hat{S}_{l_{1}}^{y, c}+\hat{S}_{l_{2}}^{y, c}\right) \hat{\tau}^{y}\right]\right. \\
& \left.+j^{z}\left(\hat{S}_{\boldsymbol{l}_{1}}^{z, c}+\hat{S}_{\boldsymbol{l}_{2}}^{z, c}\right) \hat{\tau}^{z}+\mu\left(\hat{S}_{l_{1}}^{z, c}+\hat{S}_{\boldsymbol{l}_{2}}^{z, c}\right)\right\}+\frac{1}{2}\left(\Delta_{1}^{0,1}-g \mu_{B} h^{z}\right)\left(\hat{\mathbf{1}}+\hat{\tau}^{z}\right)
\end{aligned}
$$


where, $j^{\perp}=\frac{1}{2 \sqrt{2}}, j^{z}=\frac{1}{4}$ and $\mu=\frac{1}{4}$, we use the following unitary transformation for conduction electrons in Eq. (6),

$$
\hat{\boldsymbol{S}}_{B}^{c}=\frac{1}{\sqrt{2}}\left(\hat{\boldsymbol{S}}_{l_{1}}^{c}+\hat{\boldsymbol{S}}_{l_{2}}^{c}\right), \quad \hat{\boldsymbol{S}}_{A}^{c}=\frac{1}{\sqrt{2}}\left(\hat{\boldsymbol{S}}_{l_{1}}^{c}-\hat{\boldsymbol{S}}_{l_{2}}^{c}\right)
$$

to rewrite it as

$$
\hat{H}_{1}^{e f f}=e_{0,1}-t \sum_{\langle\boldsymbol{i}, \boldsymbol{j}\rangle, \sigma}\left(\hat{c}_{\boldsymbol{i}, \sigma}^{\dagger} \hat{c}_{\boldsymbol{j}, \sigma}+h . c\right)+\tilde{j}^{\perp}\left(\hat{S}_{B}^{x, c} \hat{\tau}^{x}+\hat{S}_{B}^{y, c} \hat{\tau}^{y}\right)+\tilde{j}^{z} \hat{S}_{B}^{z, c} \hat{\tau}^{z}+\tilde{\mu} \hat{S}_{B}^{z, c}+\frac{1}{2}\left(\Delta_{1}^{0,1}-g \mu_{B} h^{z}\right)\left(\hat{\mathbf{1}}+\hat{\tau}^{z}\right)
$$

where, $\tilde{j}^{\perp}=\sqrt{2} J_{k} j^{\perp}$ and $\tilde{j}^{z}=\sqrt{2} J_{k} j^{z}$. The effective Hamiltonian given in Eq.(8) corresponds to an anisotropic single impurity Kondo Hamiltonian. Following Anderson's poor man scaling approach ${ }^{32,33}$ the effective Kondo scale can be estimated by integrating and solving two differential equations (see below) obtained from $T$-matrix scattering process of the conduction electron scattering off the pseudo spin-1/2 degree of freedom, $\tau$ :

$$
\frac{d \tilde{j}^{z}}{d \ln D}=2 \rho\left(\tilde{j}^{\perp}\right)^{2}
$$

and

$$
\frac{d \tilde{j}^{\perp}}{d \ln D}=2 \rho \tilde{j}^{\perp} \tilde{j}^{z}
$$

where $D$ is the half bandwidth cutoff and $\rho$ the density of states at the Fermi level. In the isotropic case $\tilde{j}^{\perp}=\tilde{j}^{z}=j$, the two differential Eqs. are identical and yield the Kondo scale; $\epsilon^{k} \sim e^{-\frac{1}{\rho j}}$. In the anisotropic case $\tilde{j}^{\perp} \neq \tilde{j}^{z}$ the two Eqs. (9) and. (10) give a scaling trajectory $\left(\tilde{j}^{z}\right)^{2}-\left(\tilde{j}^{\perp}\right)^{2}=$ const, and the effective Kondo scale can be obtained by the flow of the renormalised couplings along the trajectory.

For a chain of length $L$ one can write the effective Hamiltonian in terms of $\hat{\boldsymbol{S}}_{B(A)}^{c}$ as:

$$
\begin{aligned}
\hat{H}_{p}^{e f f} & =-t \sum_{\langle\boldsymbol{i , j}\rangle, \sigma}\left(\hat{c}_{\boldsymbol{i}, \sigma}^{\dagger} \hat{c}_{\boldsymbol{j}, \sigma}+h . c\right)+c J_{k} \sum_{\boldsymbol{l}}\left\{j_{\boldsymbol{l}, p}^{\perp}\left(\hat{S}_{\boldsymbol{l}, B(A)}^{x, c} \hat{\tau}^{x}+\hat{S}_{\boldsymbol{l}, B(A)}^{y, c} \hat{\tau}^{y}\right)+j_{\boldsymbol{l}, p}^{z} \hat{S}_{\boldsymbol{l}, B(A)}^{z, c} \hat{\tau}^{z}+\mu_{\boldsymbol{l}, p} \hat{S}_{\boldsymbol{l}, B(A)}^{z, c}\right\} \\
& +\frac{1}{2}\left(\Delta_{p}^{m_{1}, m_{2}}-g \mu_{B} h^{z}\right)\left(\hat{\mathbf{1}}+\hat{\tau}^{z}\right) .
\end{aligned}
$$

Here, the summation $\sum_{\boldsymbol{l}}$ goes over $\boldsymbol{l}=1, \cdots, \frac{L}{2}\left(\frac{L+1}{2}\right)$ for even(odd) $L$, and, $c$ is a normalisation factor arising from the bonding or antibonding $(B(A))$ selections of conduction electrons involved in the Kondo effect. This selection rule stems from the inversion symmetry present in the Heisenberg chain and involves pairs of sites $l\left(=1, \cdots, \frac{L}{2}\right)$ and $\boldsymbol{l}^{\prime}\left(=L-(\boldsymbol{l}-1), \cdots, \frac{L}{2}+1\right)$. One can define a site dependent effective Kondo scale of Eq. (11) by considering the flow of renormalised couplings along the scaling trajectories,

$$
\left(\tilde{j}_{l, p}^{z}\right)^{2}-\left(\tilde{j}_{l, p}^{\perp}\right)^{2}=\text { const }
$$

where $\tilde{j}_{\boldsymbol{l}, p}^{\perp}=c J_{k} j_{\boldsymbol{l}, p}^{\perp}$ and $\tilde{j}_{\boldsymbol{l}, p}^{z}=c J_{k} j_{\boldsymbol{l}, p}^{z}$. To estimate the Kondo scale we use $c=\sqrt{2}$ if two atoms at the position $\boldsymbol{l}$ and $\boldsymbol{l}^{\prime}$ are symmetrically involved in the Kondo resonance and $c=1$ if only one atom shows a Kondo resonance. The latter case corresponds for example to the central atom of an odd sized chain. Furthermore, we use a constant density of states $\rho=1$ in all cases. At a level crossing $p$ and depending on the sign of $\tilde{j}_{\boldsymbol{l}, p}^{z}{ }^{32-36}$ (see Table III) a relative estimate of Kondo scale $\left(\epsilon_{l, p}^{k} \sim e^{-\frac{1}{\rho \hat{j}_{l, p}}}\right)$ is given in Table IV.

TABLE III. Kondo scale $\left(\epsilon_{l, p}^{k} \sim e^{-\frac{1}{\rho \hat{j}_{l, p}}}\right)$ according to sign of $\tilde{j}_{l, p}^{z}$ for an anisotropic Kondo Hamiltonian.

\begin{tabular}{l|ll}
\hline \hline & $\tilde{j}_{l, p}^{z}>0$ & $\tilde{j}_{l, p}^{z}<0$ \\
\hline \hline$\left|\tilde{j}_{l, p}^{\perp}\right|>\left|\tilde{j}_{l, p}^{z}\right|$ & $\frac{1}{\tilde{j}_{l, p}}=\frac{1}{\sqrt{\left(\tilde{j}_{l, p}^{\perp}\right)^{2}-\left(\tilde{j}_{l, p}^{z}\right)^{2}}} \times \tan ^{-1}\left(\frac{\sqrt{\left(\tilde{j}_{l, p}^{\perp}\right)^{2}-\left(\tilde{j}_{l, p}^{z}\right)^{2}}}{\tilde{j}_{l, p}^{z}}\right)$ & $\frac{1}{\tilde{j}_{l, p}}=\frac{1}{\sqrt{\left(\tilde{j}_{l, p}^{\perp}\right)^{2}-\left(\tilde{j}_{l, p}^{z}\right)^{2}} \times\left[\pi+\tan ^{-1}\left(\frac{\sqrt{\left(\tilde{j}_{l, p}^{\perp}\right)^{2}-\left(\tilde{j}_{l, p}^{z}\right)^{2}}}{\tilde{j}_{l, p}^{z}}\right)\right]}$ \\
\hline$\left|\tilde{j}_{l, p}^{\perp}\right|<\left|\tilde{j}_{l, p}^{z}\right|$ & $\frac{1}{\tilde{j}_{l, p}}=\frac{1}{\sqrt{\left(\tilde{l}_{l, p}^{z}\right)^{2}-\left(\tilde{j}_{l, p}^{\perp}\right)^{2}}} \times \tanh ^{-1}\left(\frac{\sqrt{\left(\tilde{j}_{l, p}^{z}\right)^{2}-\left(\tilde{j}_{l, p}^{\perp}\right)^{2}}}{\tilde{l}_{l, p}^{z}}\right)$ & 0 \\
\hline \hline
\end{tabular}


TABLE IV. Effective Kondo scale $\left(\epsilon_{l\left(l^{\prime}\right), p}^{k} \sim e^{-\frac{1}{j_{l, p}}}\right)$ at the level crossings for two $J_{k}=2(1.5)$ values up to $\mathrm{L}=7$ using Anderson poor man scaling approach. Correspondingly, the Fig.11 shows that proper Kondo resonances appears in the QMC simulation at sites where $\epsilon_{l\left(l^{\prime}\right), p}^{k}$ dominates.

\begin{tabular}{l|llll}
\hline \hline$L=2$ & $\epsilon_{1(2), 1}^{k} \sim 0.329(0.227)$ & & & \\
$L=3$ & $\epsilon_{1(3), 1}^{k} \sim 0.346(0.243)$ & $\epsilon_{2,1}^{k} \sim 0.049(0.018)$ & & \\
$L=3$ & $\epsilon_{1(3), 2}^{k} \sim 0.113(0.055)$ & $\epsilon_{2,2}^{k} \sim 0.271(0.176)$ & \\
$L=4$ & $\epsilon_{1(4), 1}^{k} \sim 0.259(0.165)$ & $\epsilon_{2(3), 1}^{k} \sim 0.113(0.055)$ & \\
$L=4$ & $\epsilon_{1(4), 2}^{k} \sim 0.029(0.009)$ & $\epsilon_{2(3), 2}^{k} \sim 0.293(0.194)$ & & \\
$L=5$ & $\epsilon_{1(5), 1}^{k} \sim 0.251(0.159)$ & $\epsilon_{2(4), 1}^{k} \sim 0.091(0.041)$ & $\epsilon_{3,1}^{k} \sim 0.171(0.095)$ & \\
$L=5$ & $\epsilon_{1(5), 2}^{k} \sim 0.124(0.061)$ & $\epsilon_{2(4), 2}^{k} \sim 0.249(0.157)$ & $\epsilon_{3,2}^{k} \sim 0.0004(0.00003)$ & \\
$L=5$ & $\epsilon_{1(5), 3}^{k} \sim 0.007(0.001)$ & $\epsilon_{2(4), 3}^{k} \sim 0.189(0.109)$ & $\epsilon_{3,3}^{k} \sim 0.164(0.089)$ & \\
$L=6$ & $\epsilon_{1(6), 1}^{k} \sim 0.196(0.114)$ & $\epsilon_{2(5), 1}^{k} \sim 0.026(0.008)$ & $\epsilon_{3(4), 1}^{k} \sim 0.151(0.079)$ & \\
$L=6$ & $\epsilon_{1(6), 2}^{k} \sim 0.054(0.021)$ & $\epsilon_{2(5), 2}^{k} \sim 0.251(0.159)$ & $\epsilon_{3(4), 2}^{k} \sim 0.051(0.019)$ & \\
$L=6$ & $\epsilon_{1(6), 3}^{k} \sim 0.002(0.0002)$ & $\epsilon_{2(5), 3}^{k} \sim 0.113(0.055)$ & $\epsilon_{3(4), 3}^{k} \sim 0.224(0.136)$ & \\
$L=7$ & $\epsilon_{1(7), 1}^{k} \sim 0.186(0.106)$ & $\epsilon_{2(6), 1}^{k} \sim 0.062(0.025)$ & $\epsilon_{3(5), 1}^{k} \sim 0.231(0.142)$ & $\epsilon_{4,1}^{k} \sim 0.035(0.012)$ \\
$L=7$ & $\epsilon_{1(7), 2}^{k} \sim 0.103(0.048)$ & $\epsilon_{2(6), 2}^{k} \sim 0.119(0.059)$ & $\epsilon_{3(5), 2}^{k} \sim 0.012(0.003)$ & $\epsilon_{4,2}^{k} \sim 0.151(0.081)$ \\
$L=7$ & $\epsilon_{1(7), 3}^{k} \sim 0.022(0.006)$ & $\epsilon_{2(6), 3}^{k} \sim 0.214(0.128)$ & $\epsilon_{3(5), 3}^{k} \sim 0.123(0.062)$ & $\epsilon_{4,3}^{k} \sim 0.003(0.0004)$ \\
$L=7$ & $\epsilon_{1(7), 4}^{k} \sim 0.00017\left(10^{-6}\right)$ & $\epsilon_{2(6), 4}^{k} \sim 0.063(0.025)$ & $\epsilon_{3(5), 4}^{k} \sim 0.168(0.093)$ & $\epsilon_{4,4}^{k} \sim 0.108(0.052)$ \\
\hline \hline
\end{tabular}

A detailed temperature dependence of $A_{l}(\omega=0) \simeq \frac{1}{\pi} \beta G_{l}(\tau=\beta / 2)$ as a function of magnetic field and chain length is given in Fig. 11. Upon inspection, one will see that at a given critical magnetic field corresponding to a level crossing, a peak occurs at the site where the local Kondo scale dominates.

\section{Large-N mean field for the infinite Heisenberg chain of adatoms}

We consider an infinite Heisenberg chain of adatoms with periodic boundary conditions. The unit cell, $\boldsymbol{l}$, contains $n \in\left[1 \cdots N_{c}\right]$ conduction electrons $\hat{c}_{l, n, \sigma}$ and a single spin degree of freedom. In this case, we can use the identity $J_{h} \boldsymbol{S}_{\boldsymbol{l}} \cdot \boldsymbol{S}_{\boldsymbol{l}+1}=-\frac{J_{h}}{4}\left(D_{\boldsymbol{l}, \boldsymbol{l}+1} D_{\boldsymbol{l}, \boldsymbol{l}+1}^{\dagger}+D_{\boldsymbol{l}, \boldsymbol{l}+1}^{\dagger} D_{\boldsymbol{l}, \boldsymbol{l}+1}\right)$ with $\boldsymbol{S}_{\boldsymbol{l}}=\frac{1}{2} \boldsymbol{d}_{l}^{\dagger} \boldsymbol{\sigma} \boldsymbol{d}_{\boldsymbol{l}}, D_{\boldsymbol{l}, \boldsymbol{l + 1}}=\boldsymbol{d}_{\boldsymbol{l}}^{\dagger} \boldsymbol{d}_{\boldsymbol{l}+1}$ and constraint $\boldsymbol{d}_{\boldsymbol{l}}^{\dagger} \boldsymbol{d}_{\boldsymbol{l}}=1$ to define the large-N mean-field saddle point of Eq. (1)

$$
\begin{aligned}
\hat{H}_{M F}= & \sum_{n, n^{\prime}, k, \sigma} \hat{c}_{k, n, \sigma}^{\dagger} T(k)_{n, n^{\prime}} \hat{c}_{k, n, \sigma}-\frac{J_{h} \chi}{4} \sum_{\boldsymbol{l}, \sigma}\left(\hat{d}_{\boldsymbol{l}, \sigma}^{\dagger} \hat{d}_{\boldsymbol{l}+1, \sigma}+h . c .\right)-\frac{1}{2} g \mu_{B} h^{z} \sum_{l, \sigma} \sigma \hat{d}_{\boldsymbol{l}, \sigma}^{\dagger} \hat{d}_{\boldsymbol{l}, \sigma} \\
& -\frac{J_{k} V}{4} \sum_{\boldsymbol{l}, \sigma}\left(\hat{c}_{\boldsymbol{l}, 0, \sigma}^{\dagger} \hat{d}_{\boldsymbol{l}, 0, \sigma}+\text { h.c. }\right)-\lambda \sum_{\boldsymbol{l}, \sigma} \hat{d}_{\boldsymbol{l}, \sigma}^{\dagger} \hat{d}_{\boldsymbol{l}, \sigma}
\end{aligned}
$$

describing the hybridization of a band of spinons with the conduction electron. Here the mean field order parameters $V=\left\langle\hat{c}_{l, 0, \sigma}^{\dagger} \hat{d}_{l, 0, \sigma}\right\rangle$ and $\chi=\left\langle\hat{d}_{l, \sigma}^{\dagger} \hat{d}_{l+1, \sigma}\right\rangle$ have to be determined self-consistently and the Lagrange multiplier $\lambda$ enforces the constraint on average. 

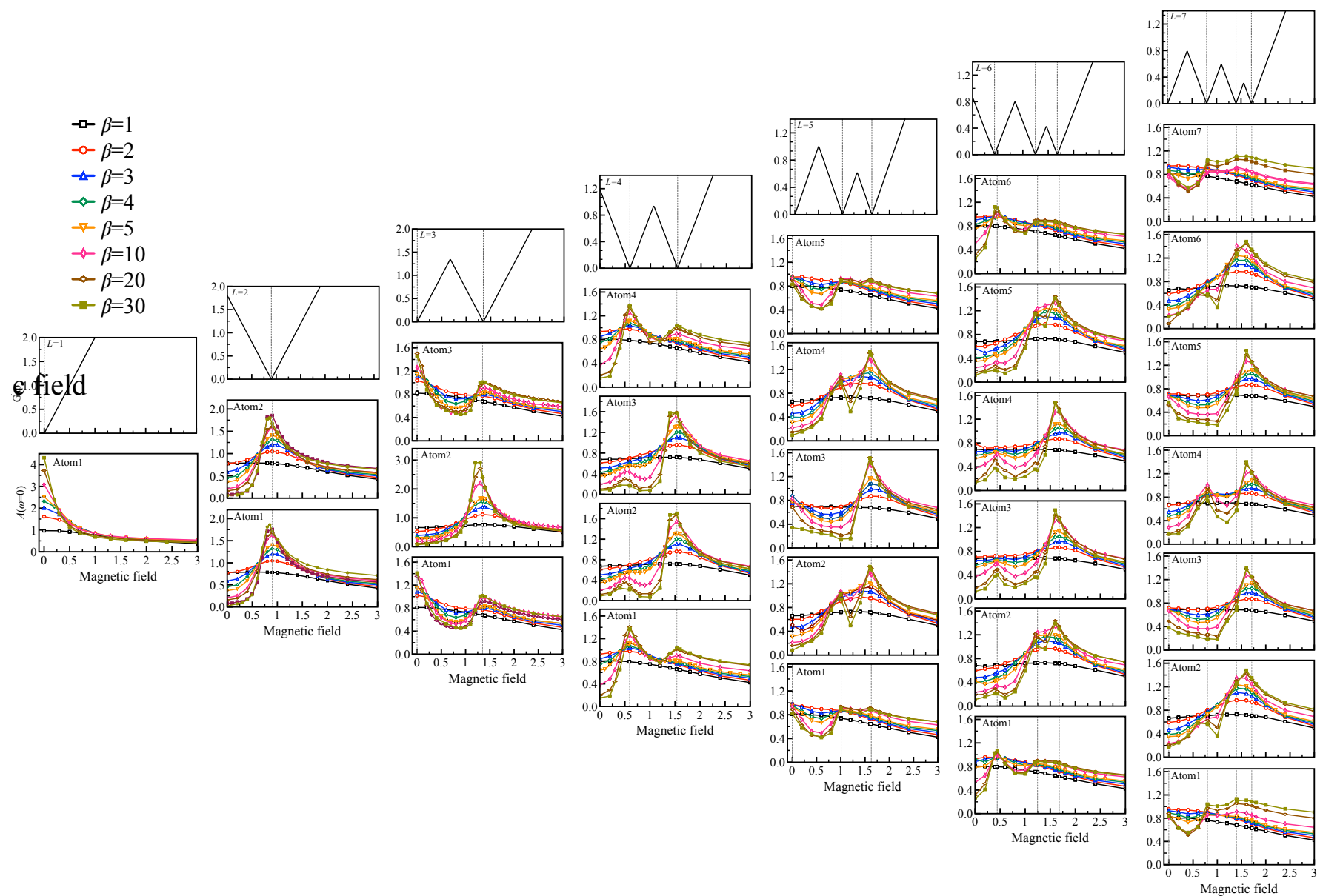

FIG. 11. QMC results for spectral function at $\omega=0$ as a function of an external magnetic field for $J_{k} / t=2, J_{h} / t=1.8$ and for different values of inverse temperature $\beta=t / k_{B} T$ up to $L=7$. This figure is directly comparable with Fig.3 reported in Ref. [11] along a cut on zero bias conductance data around $330 \mathrm{mK}$. 

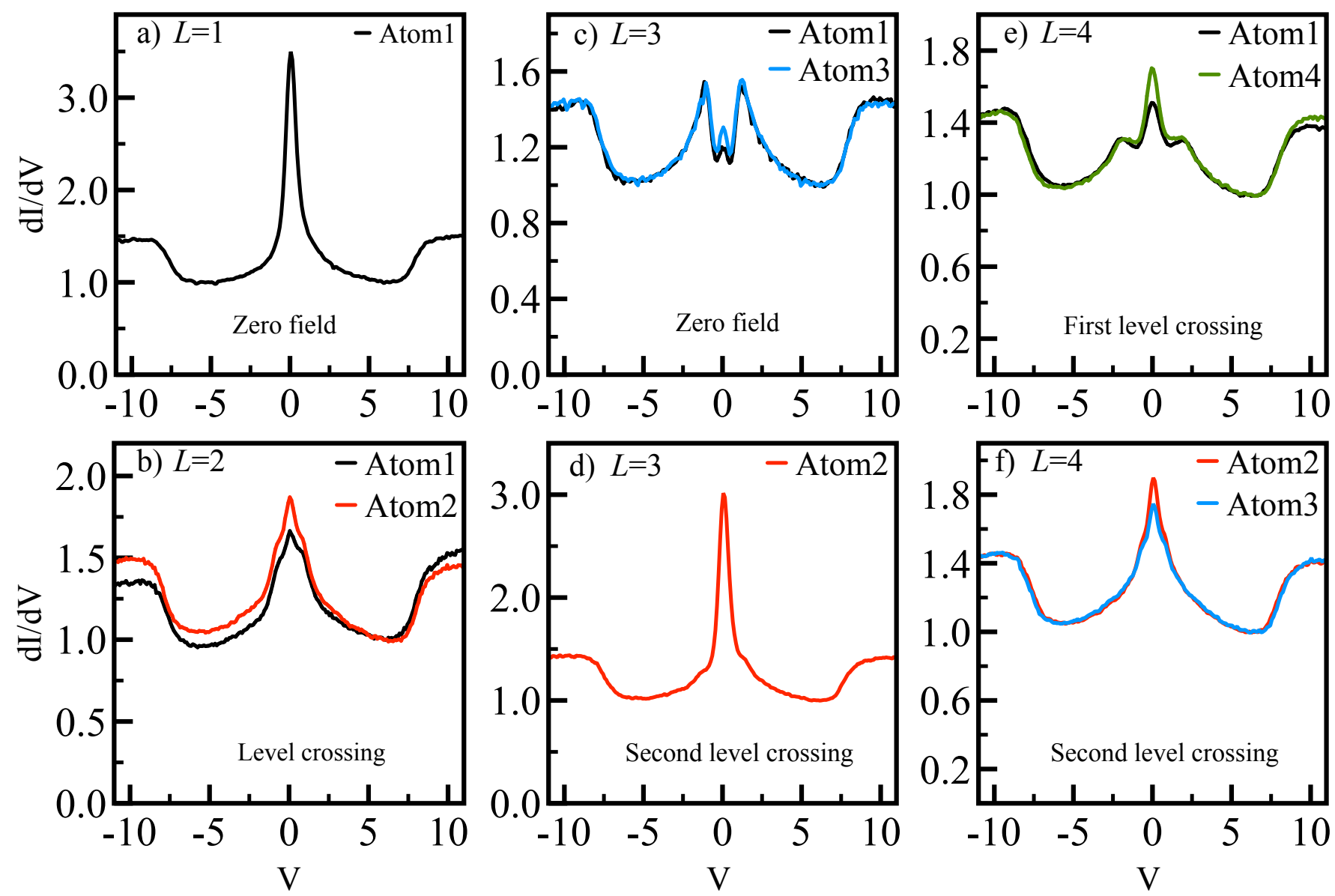

FIG. 12. Differential conductance (in atomic units) as a function of voltage measured in STM experiment of Ref. [11]. 